Meta

Journal des traducteurs

Translators' Journal

\title{
Mondialisation en cours et traduction
}

\section{Yves Gambier}

Volume 51, numéro 4, décembre 2006

La traduction des noms propres (1) et Langue, traduction et mondialisation : interactions d'hier, interactions d'aujourd'hui Language, Translation and Globalization: Interactions from Yesterday, Interactions from Today (2)

URI : https://id.erudit.org/iderudit/014347ar

DOI : https://doi.org/10.7202/014347ar

Aller au sommaire du numéro

Éditeur(s)

Les Presses de l'Université de Montréal

ISSN

0026-0452 (imprimé)

1492-1421 (numérique)

Découvrir la revue

Citer cet article

Gambier, Y. (2006). Mondialisation en cours et traduction. Meta, 51(4), 848-853. https://doi.org/10.7202/014347ar

\section{Résumé de l'article}

La mondialisation est un phénomène ambigu à la fois dans ses visées et ses conséquences. Elle est aussi diverse dans ses niveaux et domaines d'intervention, d'influence. Cinq orientations au moins peuvent lui être données. De quelle mondialisation parle-t-on donc quand on traite de traduction ? Peut-on même rapprocher mondialisation et traduction? A-t-on les moyens d'investigation ? Faute de pouvoir répondre à ces dernières questions, l'auteur propose un dialogue fictif, pour souligner certains effets et clichés au quotidien dès qu'on globalise. Le traducteur n'échappe pas aux bouleversements entrainés par les technologies de l'information et de la communication (TIC).
Ce document est protégé par la loi sur le droit d'auteur. L'utilisation des services d’Érudit (y compris la reproduction) est assujettie à sa politique d'utilisation que vous pouvez consulter en ligne.

https://apropos.erudit.org/fr/usagers/politique-dutilisation/ 


\title{
Mondialisation en cours et traduction
}

\author{
YVES GAMBIER \\ Université de Turku, Turku, Finlande \\ yves.gambier@utu.fi
}

\section{RÉSUMÉ}

La mondialisation est un phénomène ambigu à la fois dans ses visées et ses conséquences. Elle est aussi diverse dans ses niveaux et domaines d'intervention, d'influence. Cinq orientations au moins peuvent lui être données. De quelle mondialisation parle-t-on donc quand on traite de traduction? Peut-on même rapprocher mondialisation et traduction? A-t-on les moyens d'investigation? Faute de pouvoir répondre à ces dernières questions, l'auteur propose un dialogue fictif, pour souligner certains effets et clichés au quotidien dès qu'on globalise. Le traducteur n'échappe pas aux bouleversements entrainés par les technologies de l'information et de la communication (TIC).

\section{ABSTRACT}

Globalisation is an ambiguous process, both in its aims and its consequences, and varied in its levels and fields of influence. Five different meanings at least can be given to the concept. What type of globalization are we talking about when dealing with translation? Do we have any methods to link globalisation and translation? The author has doubts and therefore proposes a fictitious dialogue in order to emphasize certain daily effects and clichés regarding globalization. No translator can escape changes implied by technology (ICT).

\section{MOTS-CLÉS/KEYWORDS}

mondialisation, néolibéralisme, technologies de l'information et de la communication (TIC), libre circulation, mission du traducteur

Ceci n'est pas un exposé. Ou plutôt il ne correspondra pas sans doute aux standards et aux attentes de la plupart. Quand j'ai proposé un résumé d'intervention, le paysage mondial était encore mi-figue, mi-raisin. Depuis, c'est encore plus la loi de la jungle.

En 1998, Paul St-Pierre et Annie Brisset ont lancé un projet d'ouvrage sur traduction et mondialisation. Depuis, s'est multiplié le nombre de colloques, de publications sur l'internationalisation supposée des marchés et sur le rôle censé accru des langagiers, sans parler des ouvrages et rencontres sur la globalisation qui serait à tenir pour un fait acquis, de l'abolition prétendue des frontières, du développement des technologies de l'information et de la communication (TIC). Bref, la machine économique, financière et technologique aurait inexorablement avancé, transformant tout sur son passage, les relations internationales comme les conditions de travail, l'État-nation comme les activités culturelles, les notions de temps et d'espace comme la place et les usages des divers codes sémiotiques.

Il y a dans ces discours une fascination obsessionnelle, un simulacre monomaniaque donnant à croire que la réalité dans sa complexité se changerait par le poids des mots. Les traducteurs et les traductologues, pourtant maîtres dans les ruses de la parole, semblent aussi avoir succombé au leurre du mot «mondialisation», comme 
ils semblent parfois suivre des modes, par exemple la théorie interprétative, puis la théorie du skopos, puis la perspective à la Venuti, puis l'approche par corpus, puis...

Ainsi donc global, local, glocal, localisation, globalitaire... seraient désormais des mots-clés et remettraient en cause les idées traditionnelles de territoire, de frontière, de centre, de pouvoir, de culture, dans un monde devenu quasi automatiquement postmoderne, postcolonial, etc.

Qu'est-ce que cette mondialisation qui devait ou devrait changer nos façons de faire, de travailler, de fixer les salaires, les relations diplomatiques, commerciales, financières, culturelles, et j'en passe? Elle a des visages pour le moins contrastés:

- Elle serait pilotée en fait à partir d'un centre, au moyen d'une politique prenant appui sur la force militaire (le budget américain de la défense pour 2003 dépassait les dépenses militaires de tous les pays industrialisés, Russie et Chine incluses).

- Elle permettrait des scandales du type Enron ou Worldcom ou Vivendi.

- Elle serait un unilatéralisme hégémonique autorisant certains à ne pas accepter les contrôles des armes chimiques, biologiques et nucléaires, le traité sur les mines antipersonnel, à ne pas reconnaître la justice internationale via la Cour pénale internationale, à rejeter les accords de Kyoto sur la limitation des gaz à effet de serre, à voter des lois d'extraterritorialisation (contre Cuba, la Syrie, la Lybie), à augmenter les tarifs douaniers quand cela arrange les affaires nationales, à ignorer les conventions sur les droits de l'enfant (1989), sur l'élimination des formes de discrimination contre les femmes (1979), sur les droits économiques, sociaux et culturels (1966), entre autres exemples.

Je m'éloigne du sujet de cette publication? Mais non: si on ne met pas de la chair ou un essai de définition sur «mondialisation », la discussion n'est pas possible. Si on ne reconnaît pas que le concept est un piège qui désigne en fait à la fois l'imposition d'une économie néolibérale, largement dominée par les États-Unis, et la projection imaginaire (idéologique) d'un monde sans frontières, on se condamne à la gentille spéculation ou au mieux au délire prospectif. L'interdépendance accélérée a pu faire croire dans la décennie 1990 que la seule économie et peut-être la technologie constituent des facteurs fondamentaux. Les actualités de ces derniers mois sont venues nous rappeler que la politique, l'idéologie et le militaire étaient aussi décisifs. Les guerres (civiles, préventives, contre-révolutionnaires, frontalières, nationales, claniques, ethniques, de sécession, culturicides) seraient chassées du tragique pour être réglées sous l'autorité de juges autoproclamés maîtres du bien et du mal. Démocratie, justice, liberté, droits seraient devenus des mots de passe de cette mondialisation violente. Peut-on vraiment deviser comme si ces mots volés étaient toujours partagés, comme si la critique n'était pas vite diabolisée?

La mondialisation (libérale) avec sa division du travail connaît une extension dans ses systèmes de communication, dans son économie, dans quelques valeurs uniformisantes (par exemple par le moyen des industries culturelles du cinéma, de la chanson, de la télévision) et aussi dans son underground (mafia criminelle). Mais il lui manque une gouvernance collective, d'où la balkanisation et les recolonisations qu'elle entraîne, le contrôle de ses pouvoirs, une conscience commune d'appartenance, un droit respecté. Il lui manque aussi une mémoire qui lui ferait saisir qu'elle n'est pas nouvelle, mais issue d'un long processus historique, avec ses contradictions. La première "économie-monde» est concomitante des conquêtes européennes du $\mathrm{XVI}^{\mathrm{e}}$ s., à partir des réseaux commerciaux portugais et espagnols, puis hollandais et anglais. La seconde moitié du XviII ${ }^{\mathrm{e}}$ s. esquisse le «droit cosmopolitique» (Kant). 
De quelle mondialisation parle-t-on quand le moindre éternuement propagerait un virus inconnu (SRAS, grippe aviaire) et paralyserait tous les ateliers d'une région? De quelle mondialisation parle-t-on quand la libre circulation des marchandises, des capitaux, des services serait garantie tandis que la circulation des personnes serait soumise au plus strict des contrôles? Une des activités les plus lucratives aujourd'hui, ce n'est pas la communication interculturelle, interlinguistique, mais la sécurité, c'està-dire le conseil, la formation, le soutien logistique, la fourniture de personnel et de moyens dans les domaines de la surveillance, et du gardiennage, le croisement de banques de données, pour protéger certains biens et réguler les comportements, rendre dociles. De quelle mondialisation parle-t-on après les crises financières en Asie (1997-1998), les secousses en Argentine, en Indonésie, au Sénégal et dans tous les pays endettés? Ne pas répondre à cette simple question, c'est se faire les chantres, les zélateurs d'une certaine mondialisation basée sur la compétitivité posée comme vertu suprême au détriment de l'emploi, du salaire, de la protection sociale, basée sur la privatisation, ignorante de l'intérêt général, basée sur le marché qui serait tout puissant et infaillible, basée sur la stabilité monétaire à n'importe quel prix, basée enfin sur la soi-disant libération des échanges, supposée favorable à tous, en dépit de la polarisation des investissements dans les régions déjà les plus riches, en dépit des taxes douanières imposées comme un yoyo selon les intérêts en jeu. Bref, ne pas répondre à la question, ce serait légitimer bien des idées reçues de la doxa libérale (sur les «nécessaires» privatisations, y compris celles de l'éducation, sur le «poids» de l'État, sur les minima sociaux, sur la flexibilité du marché du travail et autres credo). Ce serait naïvement croire que les traducteurs sont hors des circuits et des enjeux de la marchandisation actuelle, que la situation est irréversible, que le «dialogue des cultures» a cédé la place irrémédiablement au «choc des civilisations».

On peut parler de la mondialisation dans au moins cinq directions différentes, non antagonistes:

- comme simple internationalisation des échanges, entre autres commerciaux (le phénomène ne serait alors ni nouveau ni récent);

- comme étape du développement économique néolibéral, avec son extrême caricatural, la macdonaldisation;

- comme tentative d'intégrations régionales, en Europe, entre les Amériques, en Asie du Sud-est, souvent plus poussée par la promotion du libre-échange que par une certaine vision politique, une telle démarche s'intégrant donc dans le développement signalé ci-dessus;

- comme gestion de l'ensemble des «biens communs de l'humanité» (culture, éducation, santé, environnement, eau, notamment);

- comme réseau tissé par les technologies de l'information et de la communication (TIC) permettant le jeu entre global et local et comportant des implications sur la production, la distribution, la circulation des documents, sur l'organisation du travail, entre autres.

Mondialisation et traduction? Les traducteurs travaillent pour la globalisation des biens, des brevets, des services, pour l'industrie de l'électronique, les pharmaceutiques, les biotechnologies, etc. - plus que pour la libre circulation des personnes. Ils offrent leurs services, bien normalement, à ceux qui payent, au détriment parfois de leur éthique. Mais y a-t-il vraiment choix entre traduction pour l'industrie agroalimentaire et traduction pour la protection de l'enfance? Entre traduction pour 
l'industrie aéronautique et traduction à propos de l'environnement dégradé? Entre interprétation pour le policier qui expulse l'étranger et interprétation pour le réfugié qui cherche asile? Peut-on ne pas être complice de l'ordre dominant qui refoule ses contradictions et tente de plus en plus de contrôler les paroles différentes, de vassaliser, de domestiquer? Peut-on faire autre chose que sous-titrer films et émissions télévisées de plus en plus formatés (synonyme d'internationalisation)?

Mondialisation et traduction? Quoi en dire et comment, quand on n'a pas de statistiques sur les communications multilingues, à part les rapports Ovum (1995), sans outils de recherche pour décrire les possibles processus d'extension et d'intensification de l'interdépendance, de l'intégration comme de la fragmentation, et qu'il reste à savoir à quel concept, quel sens, quelle symbolisation du monde on renvoie? Et cela vaut aussi pour l'altermondialisation ou l'antiglobalisme. Est-ce que le nom même de «mondialisation» désigne après tout quelque chose pour ou contre quoi on puisse prendre position? Quel lien entretient-il avec globalisation? Quoi en dire et comment quand on ne connaît pas les volumes des besoins, de la demande, de l'offre? Quand on n'a pas de graphiques indiquant les flux d'échange? Dans le rapport 2002 de l'ONU sur le développement dans le monde arabe, il est dit qu'aujourd'hui, cette entité traduit au total moins de livres étrangers que la seule Grèce. La traduction deviendrait-elle indice de développement? Quoi en dire et comment quand on n'a pas d'indicateurs fiables à comparer dans le temps? Quand on n'a pas de centres de production territorialement délimitables? Peut-on alors parler de concentration, de délocalisation, de sous-traitance? On peut supputer, esquisser des modèles, élaborer des discours politico-linguistiquement corrects (affirmant que toutes les langues sont égales, qu'elles peuvent exprimer tout le savoir, que les langues minoritaires ont droit à une reconnaissance officielle, que les langues menacées doivent être protégées, que perdre sa langue c'est perdre ses racines). Peut-on cependant même tracer les lignes d'une politique traductionnelle sans élaborer une géostratégie des langues, des politiques éditoriales, médiatiques, communicationnelles, éducatives?

Voici pourquoi ce texte n'est pas un exposé: il n'a ni les moyens ni les outils pour dresser un bilan, un plan. Au lieu d'une présentation plus ou moins objective, factuelle, hypothético-déductive, et après un effort pour dire mon malaise face à la mondialisation (comme concept) c'est-à-dire d'où je parle, je voudrais me tourner vers la fiction. Oui, la fiction, moyen d'exploration aussi. Elle permet d'exprimer paradoxes et contradictions, au contraire d'un certain discours universitaire astreint à la non-contradiction, à la cohérence rigide. La mondialisation est en cours, à des vitesses et des résistances variables ici et là. Elle ne cesse de brouiller mes repères, d'accroître mon désarroi. Le dialogue fictif que je vous propose souligne la dimension technologique du devenir actuel et voudrait dire ce qui est vécu, appréhendé au quotidien, sans autre prétention (ni scientifique ni littéraire).

La scène se passe au téléphone, à moins que ce soit devant un kiosque à traductions, comme il existe des kiosques à journaux ou à saucisses, entre (a) un commanditaire ou donneur d'ouvrage qui vend des jouets et (b) un salarié d'une agence de traduction. Ce dernier a, me semble-t-il, le beau rôle.

(a) Allo! J'ai une traduction à faire faire, pour assez vite. Pourriez-vous me rendre ce service?

(b) Ça dépend des ingrédients que vous voulez avec?

(a) Pardon? 
(b) Oui, quelle sorte de traduction souhaitez-vous?

(a) Eh! bien (irrité), une traduction! Mon catalogue est en estonien et je le voudrais en anglais, français, allemand, espagnol et japonais! C'est pas difficile!

(b) Ah! bon! Donc il n'y a pas de photos, de graphiques, de tableaux?

(a) Euh!

(b) C'est un transfert interlinguistique que vous voulez? Mais dites-moi, que recherchez-vous exactement?

(a) Je ne vous suis pas! Bien sûr qu'il y a des images et des schémas de montage pour certains jouets extérieurs! Mais c'est pas ce que je vous demande...

(b) Vous voulez dire qu'images et schémas ne font pas partie du catalogue?

(a) Si! Si!

(b) Voyez-vous, si vous voulez votre catalogue comme imprimé, cédérom ou même site web, les trois versions doivent être adaptées.

(a) Je veux le texte en cinq langues!

(b) Sur le même document ou en documents séparés?

(a) Ça fait une différence?

(b) Oui, justement...

(a) Mais une traduction, c'est une traduction!

(b) Eh! bien! Dites moi, cher Monsieur, votre traduction, vous la voulez libre, éditée, acceptable, dynamique?

(a) Je croyais que vous saviez ce qu'était une traduction! Vous êtes quand même une agence spécialisée! D'ailleurs, est-ce que je ne pourrais pas parler à l'un de vos traducteurs directement?

(b) Je n'ai pas de traducteurs sous la main. Ils travaillent, certains à des milliers de kilomètres d'ici, vous savez.

(a) Ben, ben...d'accord. Mais n'empêche, vous devriez savoir ce qu'est une traduction!

(b) Oui, voilà pourquoi justement je voudrais savoir à qui vous destinez votre catalogue? À des grossistes? Des marchands de jouets au détail? Des crèches et écoles maternelles?

(a) C'est notre département de marketing et export qui s'occupe de ça!

(b) Certainement. Mais votre document ne va-t-il pas accompagner les jouets? Ou le voulez-vous comme document pour promouvoir vos ventes?

(a) C'est pour vendre davantage, alors que la concurrence est de plus en plus dure, avec les Chinois et les Thaillandais qui ne payent pas leurs ouvriers!

(b) D'accord, d'accord! Ne pensez-vous pas alors que ce catalogue pourrait aussi être diffusé sur d'autres supports que le papier? Nous pouvons vous offrir ces services.

(a) Au même prix que la traduction?

(b) Traduction, transposition, localisation, adaptation... Nous pouvons vous offrir un document prêt à remplir les fonctions que vous lui assignez, sur les supports appropriés!

(a) Charabia! Je vous laisse mon texte et vous me donnez tous les mots, sans omission ni calques, en anglais, en français, en allemand, en espagnol et en japonais!

(b) Vous voulez dire que vous souhaitez obtenir une traduction juxtalinéaire où vous pouvez repérer tel ou tel terme dans les cinq langues?

(a) Je veux augmenter nos ventes, parbleu!

Vous savez, il y a des possibilités de traduction gratuite sur le Net...comme il pourrait y avoir des distributeurs automatiques de plateaux-repas dans les avions. (silence momentané, l'agacement est perceptible de part et d'autre)

(b) Bon, votre traduction, faut-il lui ajouter du ketchup, vous l'envelopper?

(a) Ah! Mais je ne comprends pas..., vous faites le difficile! Si ça continue, je vais chercher une autre agence... 
(b) ... et on vous posera les mêmes questions, si on vous propose de la qualité! Voyezvous, comme un sandwich qui peut être habillé de différentes salades et assaisonnements, votre traduction peut être plus ou moins brute, adaptée à vos clients!Vous savez les Japonais n'ont pas les mêmes goûts, les mêmes attentes que les Allemands! Vous pouvez la lire sur place ou l'emporter!

(a) Avec les langues choisies, nous visons différents marchés - anglophone, francophone...

(b) Justement! C'est l'anglais des États-Unis, des Indes, de Grande-Bretagne, d'Australie... que vous recherchez?

(a) Balivernes! L'anglais, c'est l'anglais!

(b) Pas tout à fait, mon bon Monsieur! À propos, votre catalogue en estonien est-il long?

(a) Il n'existe pas encore! On voudrait le rédiger, le composer quasi en même temps que vous le traduirez.

(b) Pas de problème, mais une fois encore, pourriez-vous me dire si vous voulez notre travail mis en page, avec des produits dérivés comme un $\mathrm{CD}$, une bande-annonce sur téléphone mobile?

(a) Je vais y réfléchir. Auparavant, qu'en est-il de vos prix?

(b) Cela dépend de vos exigences quant à la forme finale que vous souhaitez, aux supports. Nous pouvons aussi imprimer à la demande le nombre d'exemplaires désirés.

(a) Oui, oui... pourquoi pas me vendre aux enchères votre offre de traduction sur le Net?

(b) C'est ce que nous faisons! D'ailleurs, puis-je vous signaler aussi que vous pouvez passer commande, décrire votre cahier des charges sur notre site Web, sans nécessairement nous téléphoner!

(a) Ah bon! Ce que je veux, c'est une traduction. Vite et bien faite!

(b) À votre service, Monsieur $24 \mathrm{~h}$ sur 24, 7 jours sur 7! Ah! J'oubliais... en achetant nos services, vous permettez aux enfants de l'école St-Jérôme de s'acheter un filet de volley-ball.

Le silence est retombé, troublant sans doute les représentations de la traduction chez le vendeur de jouets et ne rassurant guère le salarié de l'agence de traduction. On a ici un double paradoxe: tandis que le premier continue de mettre au second plan, sinon à dévaloriser, ses problèmes de communication multilingue alors qu'il vise des marchés de plus en plus diversifiés, le second peine à faire admettre les transformations de sa pratique professionnelle alors qu'il y a peu, il était facilement accusé de rester retranché derrière ses piles de dictionnaires. C'est ainsi que va la «mondialisation », entre intérêts à court terme et métamorphoses des conditions de travail, entre déclarations triomphalistes et tensions des relations - encore loin de la société civile internationale pacifiée par le droit et l'harmonie. 